\title{
Anterolateral papillary muscle rupture as a rare complication of unusual infective endocarditis
}

\author{
Salma Charfeddine ${ }^{1}$, Syrine Triki ${ }^{1}$, Majdi Gueldiche ${ }^{2}$, Tarak Ellouze ${ }^{1}$, Faten Triki $^{1}$, and \\ leila Abid ${ }^{1}$ \\ ${ }^{1}$ Hedi Chaker Hospital \\ ${ }^{2}$ Habib Bourguiba Hospital
}

November 11, 2020

\begin{abstract}
The rupture of mitral papillary muscles is a very rare complication of the infective endocarditis. We report a case of anterolateral papillary muscle rupture resulting in severe mitral regurgitation, due to infective endocarditis in a young man without previous heart disease.
\end{abstract}

Anterolateral papillary muscle rupture as a rare complication of unusual infective endocarditis Salma Charfeddine ${ }^{a}$, Syrine Triki ${ }^{a}$, Majdi Gueldiche ${ }^{b}$, Tarek Ellouze ${ }^{a}$, Faten Triki ${ }^{\text {a }}$, Leila Abid $^{\mathrm{a}}$

Department of cardiology, Hedi Chaker University Hospital of Sfax. University of medicine of Sfax Faculty of medicine of Sfax, Tunisia

Department of cardiovascular surgery, Habib Bourguiba University Hospital of Sfax. University of medicine Faculty of medicine of Sfax, Tunisia

Corresponding author: Salma Charfeddine

Phone number: +21698638435

Email address:selma_charfeddine@yahoo.fr

Short title: papillary muscle rupture and infective endocarditis

\section{Introduction}

Infective endocarditis (IE) is not a rare disease (1). It is more frequent in the presence of valvular disease (1). The IE lesions consist on vegetations and valvular destruction that are located essentially on the valves. The rupture of the mitral papillary muscle due to IE is a very rare complication $(2,3)$. We report here the case of severe mitral regurgitation occurring on normal mitral valve with anterolateral papillary muscle rupture due to IE.

\section{Case presentation}

A diabetic 26-year-old man was admitted to our center due for prolonged fever. He had no history of rheumatic or congenital heart disease. He had been well until three weeks ago, when he began to have fatigue and general malaise, as well as loss of appetite. At admission, the patient was febrile at $39^{\circ} \mathrm{C}$ and tachycardic. His lung sounds were clear, and no cardiac murmur was audible. There were no signs of heart failure. 
The laboratory tests showed an inflammatory syndrome. An echocardiographic evaluation revealed a normal global systolic left ventricular function, no valvular disease but we noted a $15 \times 11 \mathrm{~mm}$ mobile oscillating large mass with anechogenic content attached to the mitral anterolateral papillary muscle (Figure 1 ).

Despite the intravenous antibiotic therapy, fever had persisted. A thoraco-abdominal computed tomography revealed multiple hypodensities of the brain, the liver and the spleen consistent with septic emboli. The usual blood cultures were negative and these results were attributed to prior antibiotics. Few days after hospitalization, the patient's clinical condition worsened and pulmonary congestion appeared suddenly. The physical examination revealed a new pansystolic murmur consistent with mitral regurgitation. There was no electrocardiographic change suggesting an acute or subacute myocardial infarction.

Transthoracic echocardiography (TTE) and transesophageal echocardiography (TEE) examinations were repeated. Echocardiographic studies showed a hyperdynamic left ventricle with no regional wall-motion abnormality but we noticed severe mitral regurgitation with a prolapse of the anterior mitral valve due to the rupture of the mitral anterolateral papillary muscle, which explained the patient clinical status (Figure $\mathbf{2}$ ). The diagnosis of IE complicated by severe mitral regurgitation was established. The patient was admitted to intensive care unit for stabilization with medical therapy. Then, he underwent urgent prosthetic mitral valve replacement. On surgical inspection, total complete rupture of the tip of anterolateral papillary muscle was found (Figure 3 ).

The histologic examination of the resected tissues revealed a non-specific inflammation with fibrosis. The valve culture was negative. Given the histologic examination results, the diagnosis was a mitral valve IE complicated by anterolateral papillary muscle rupture due to direct germ invasion. After 8 weeks of IV antibiotic therapy, the patient was uneventfully discharged.

\section{Discussion}

IE is a commonly encountered clinical problem, especially in the patients with predisposing heart disease (1). Most often, the mitral valve regurgitation in IE is due to the destruction of the valvular leaflets themselves. The isolated rupture of the antero-lateral papillary muscle is much rarer and has only been described to the best of our knowledge in four previous cases $(3-6)$. There are many possible causes of ruptures of papillary muscle in IE that include ischemic necrosis due to coronary embolism (7), deposition of bacteria due to aortic regurgitation (8), or direct invasion along the sub-valvular apparatus by virulent germ like staphylococcus (9). In our case, the cause of mitral regurgitation was due to direct germ invasion of the papillary muscle. The transesophageal echocardiography (TEE), with the trans-gastric view is essential to determine the mechanism of mitral regurgitation in such cases (10). The ruptured anterolateral papillary muscle is well-defined as a separate mass attached to the chordae (10). Although we were unable to show any bacteria on histological examination and culture of the papillary muscle, active inflammation was a strong criterion for the IE diagnosis. The absence of isolated germ may be explained by the use of empiric antibiotics in our patient at admission.

In conclusion, if a huge mass is observed attached to the mitral apparatus on echocardiography in the case of sepsis, the diagnosis of IE should be made even in the absence of valvular disease and the rupture of the papillary muscle in the setting mitral regurgitation should be considered.

\section{Acknowledgments: None}

\section{Conflict of interest: None}

\section{References}

1. Habib G, Lancellotti P, Antunes MJ, Bongiorni MG, Casalta J-P, Del Zotti F, et al. 2015 ESC Guidelines for the management of infective endocarditis: The Task Force for the Management of Infective Endocarditis of the European Society of Cardiology (ESC). Endorsed by: European Association for Cardio-Thoracic Surgery (EACTS), the European Association of Nuclear Medicine (EANM). Eur Heart J. 21 nov 2015;36(44):3075128. 
2. Gouda P, Weilovitch L, Kanani R, Har B. Case report and review of nonischemic spontaneous papillary muscle rupture reports between 2000 and 2015. Echocardiography. mai 2017;34(5):786-90.

3. Whitehead NJ, Li S, Lai K. Anterolateral papillary muscle rupture in Staphylococcus aureus endocarditis due to direct bacterial invasion of papillary muscle. Echocardiography. sept 2017;34(9):1382-4.

4. Maruo T, Komiya T, Shimamoto T, Kadota K, Mitsudo K. Spontaneous papillary muscle rupture with localized endocarditis. Eur Heart J Cardiovasc Imaging. janv 2015;16(1):115.

5. Nurkalem Z, Gorgulu S, Orhan AL, Demirci DE, Sargin M, Gumrukcu G. Papillary muscle rupture secondary to infective endocarditis. Echocardiography. sept 2008;25(8):901-3.

6. Najib MQ, Lee HR, DeValeria PA, Vinales KL, Surapaneni P, Chaliki HP. Anterolateral papillary muscle rupture: an unusual complication of septic coronary embolism. Eur J Echocardiogr J Work Group Echocardiogr Eur Soc Cardiol. fevr 2011;12(2):E10.

7. Sugimoto T, Shimanuki T, Minowa T, Uchino H, Nakamura C. [A case report of infective endocarditis with total rupture of the posterior papillary muscle after aortic valve replacement]. Kyobu Geka. dec 1998;51(13):1120-2.

8. Moorjani N, Saad R, Gallagher P, Livesey S. Endocarditis of the mitral valve posteromedial papillary muscle. J Card Surg. mars 2014;29(2):213-5.

9. Terai H, Okada Y, Hamaya H, Sugiki K, Ohno T. [Successful surgical treatment in a case of complete rupture of the posterior papillary muscle of the mitral valve caused by infective endocarditis]. Zasshi J Nihon Kyobu Geka Gakkai. juill 1994;42(7):1101-4.

10. Kim MY, Park CH, Lee JA, Song JH, Park SH. Papillary Muscle Rupture after Acute Myocardial Infarction. Korean J Intern Med. dec 2002;17(4):274-7.
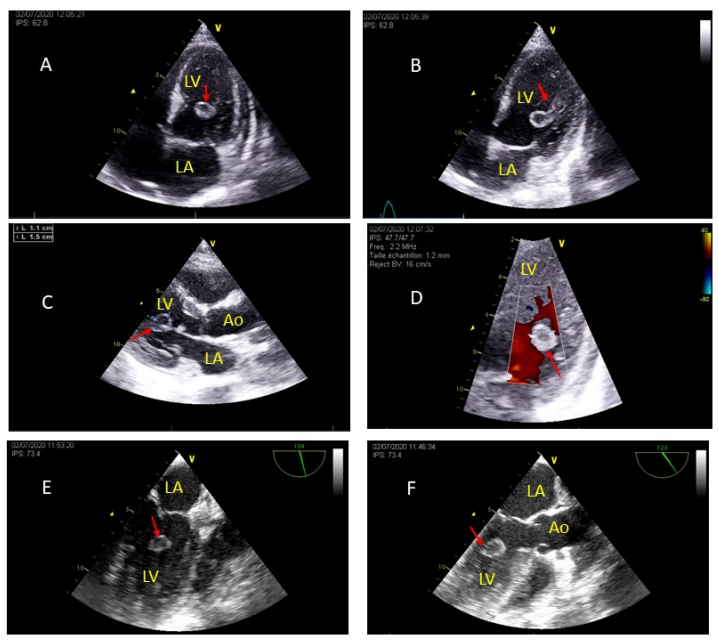

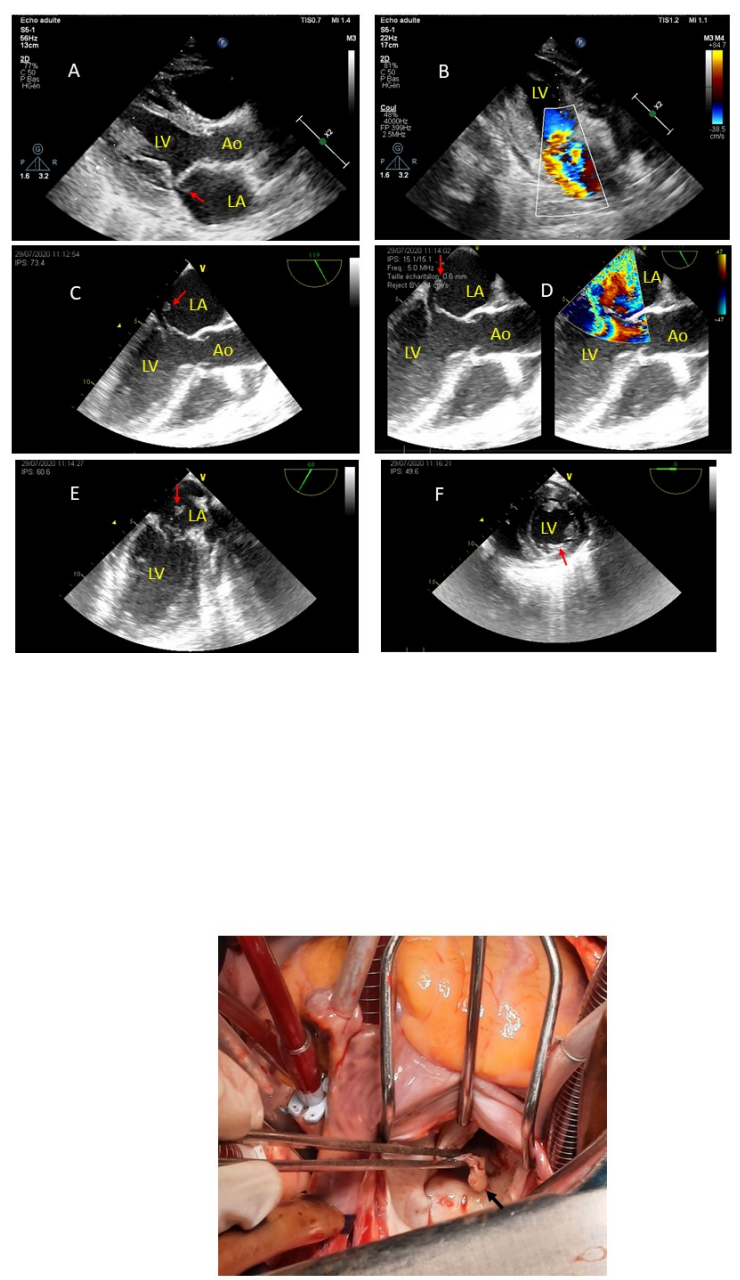